\title{
Interação entre medicamentos antidepressivos e álcool em estudantes universitários
}

\author{
Interaction between antidepressant drugs and alcohol in university students \\ Interacción entre drogas antidepresivas y alcohol en estudiantes universitarios
}

Recebido: 28/10/2021 | Revisado: 13/11/2021 | Aceito: 14/11/2021 | Publicado: 24/11/2021

\author{
Millena Sayuri Yoshida \\ ORCID: https://orcid.org/0000-0002-6828-5351 \\ Centro Universitário da Fundação Assis Gurgacz, Brasil \\ E-mail: msyoshida@minha.fag.edu.br \\ Ana Claudia Cabral dos Santos Reis \\ ORCID: https://orcid.org/0000-0002-9661-3834 \\ Centro Universitário da Fundação Assis Gurgacz, Brasil \\ E-mail: anaclaudia@fag.edu.br
}

\begin{abstract}
Resumo
A utilização de fármacos associados a substâncias alcoólicas trata-se de um problema, ao qual os profissionais de farmácia deparam-se constantemente, tal problemática é acentuada ainda mais quando o medicamento tem intuito antidepressivo, posto que as interações podem apresentar danos, desde moderados a graves e até mesmo óbitos. De modo geral, através de uma pesquisa bibliográfica descritiva, baseada em 10 artigos científicos cujos materiais foram disponibilizados nas plataformas Scielo, Pubmed, Google Acadêmico e Periódicos CAPES, buscou-se evidenciar considerações pertinentes ao uso de fármacos associados ao álcool na população universitária. Os resultados da pesquisa apontaram que grande parte dos acadêmicos passaram a fazer uso de fármacos antidepressivos após o ingresso na universidade, o que demonstra um relativo adoecimento da comunidade acadêmica, gerado pelo estresse, pela cobrança, pela sobrecarga física e mental, entre outros fatores, assim, os autores evidenciam acerca dessa comunidade, a grande evidência do uso de álcool e de outras substâncias, que quando associadas aos antidepressivos podem causar consequências desastrosas. Percebe-se que há um certo conhecimento e conscientização dos estudantes sobre os efeitos colaterais da utilização de medicamentos com bebidas alcoólicas, contudo, essas evidências não se mostraram eficientes para que houvesse a minimização da prática. Faz-se necessário o desenvolvimento de mais estudos por parte da comunidade científica, a fim de desvendar e expor à sociedade maiores contribuições sobre a temática, de modo a evitar os impactos gerados por essa prática.
\end{abstract}

Palavras-chave: Ingestão de álcool; Antidepressivos; Universitários.

\begin{abstract}
The use of medication associated with alcoholic substances is a problem, which pharmacy professionals are constantly faced with. This problem is even more accentuated when the medication has an antidepressant purpose, as interactions can present damage, from moderate to even Deaths. In general, through a descriptive bibliographic research, based on 10 scientific articles, we sought to highlight the important considerations regarding the use of medication associated with alcohol in the university population. The survey results showed that most students started using antidepressant medication after entering university, which demonstrates a relative illness in the academic community, generated by stress, demand, physical and mental overload, among other factors, thus, the authors highlight about this community, the great evidence of the use of alcohol and other substances, which, associated with antidepressants, can cause disastrous consequences. It is noticed that there is a certain knowledge and awareness of students about the effects of using medication with alcoholic beverages, however, this evidence does not apply to efficiency so that there was a minimization of the practice. The development of more studies by the scientific community is necessary, in order to unveil and expose to society greater contributions on the subject, in order to avoid the impacts generated by this practice.
\end{abstract}

Keywords: Antidepressants; Alcohol intake; College students.

\section{Resumen}

La utilización de fármacos asociados a las sustancias alcohólicas se trata de un problema del cual los profesionales de farmacia ven constantemente, tal problemática es acentuada aún más cuando el medicamento tiene una labor antidepresiva, ya que las interacciones pueden presentar daños que van de moderados a graves e incluso la muerte. De 
una manera general, mediante una investigación bibliográfica descriptiva, basada en diez ensayos científicos, se buscó evidenciar consideraciones pertinentes al uso de fármacos al alcohol en la población universitaria. Los resultados de la investigación señalaron que gran parte de los académicos pasaron a utilizar fármacos antidepresivos después del ingreso en la universidad, lo que demuestra que es una enfermedad relativa a la comunidad académica, generada por el estrés, las exigencias, la sobrecarga física y mental, entre otras, entonces, los autores evidencian acerca de esa comunidad, la gran prueba del uso de alcohol y de otras sustancias, que cuando asociadas a los antidepresivos pueden tener consecuencias desastrosas. Se puede percibir que hay un cierto conocimiento y concientización de los estudiantes sobre los efectos colaterales del uso de medicinas con bebidas alcohólicas, sin embargo, estas pruebas no se muestran eficientes para que hubiese la disminución de tal práctica. Es necesario el desarrollo de más estudios por parte de la comunidad científica, a fin de desvendar y exponer a la sociedad mayores contribuciones sobre el tema, para evitar los impactos generados por esa práctica.

Palabras clave: Antidepresivos; Ingestión de alcohol; Universitarios.

\section{Introdução}

Existem no mercado farmacêutico atualmente várias classificações de fármacos antidepressivos e sua escolha deve ser baseada na eficácia do medicamento de acordo com as características dos sintomas do transtorno, efeitos adversos e histórico pessoal e familiar do paciente (Neves, 2015). Os antidepressivos são classificados em inibidores da monoaminoxidase (IMAOs), antidepressivos tricíclicos (ADTs), inibidores seletivos da recaptação de serotonina (ISRSs) e inibidores seletivos da recaptação da serotonina e noradrenalina (ISRSNs) (Rang et al., 2012).

Os antidepressivos têm como principal objetivo inibir a recaptação de determinados neurotransmissores e/ou diminuir a sua destruição através do funcionamento de uma enzima chamada monoaminoxidase, que resulta em um aumento no nível de neurotransmissores na fenda sináptica (Cunha \& Gandini, 2009).

Os primeiros fármacos utilizados no tratamento da depressão foram os inibidores da monoaminoxidase (IMAOs), porém atualmente foram substituídos por outras classes de antidepressivos pelo fato de estes possuírem menos efeitos adversos e melhores eficácias clínicas. A fenelzina, tranilcipromina e a iproniazida são os principais medicamentos que fazem parte dessa classe de fármacos (Rang et al., 2012). Os IMAOs inibem a MAO, enzima responsável por metabolizar serotonina, adrenalina, noradrenalina e dopamina, aumentando o nível de neurotransmissores nos neurônios no sistema nervoso central e simpático, por consequência obtendo o efeito terapêutico para depressão (Neves, 2015). Apesar da eficácia clínica, o uso de IMAOs associados ao consumo de alimentos que possuam tiramina em sua composição faz com que ocorra um acúmulo dessa substância nas terminações nervosas que liberam adrenalina e norepinefrina causando uma crise hipertensiva (Goodman \& Gilman, 2012).

Alguns dos efeitos adversos podem ocorrer caso haja uma estimulação central excessiva, como por exemplo tremores, excitação e insônia. Alguns outros efeitos que devem ser levados em consideração como aumento de apetite, ganho de peso e em superdosagem convulsões, podem ser considerados extremos e a administração do fármaco deve ser interrompida (Rang \& Dale, 2011).

O indivíduo que ingere bebidas alcoólicas com frequência, busca enxergar o mundo de outra forma alterando sua percepção e aliviando sentimentos ruins sobre os outros ou sobre si próprio (Griffith, 1995). O uso exacerbado de bebidas alcoólicas pode ser motivado por elevados níveis de estresse psicológico, ansiedade, depressão e baixa autoestima (Masur \& Monteiro, 1983). Alguns estudos com estudantes revelaram que, para eles, o consumo de álcool estaria relacionado como uma solução para fugir dos problemas cotidianos, de situações negativas em geral, pressão social e, principalmente, pelo prazer da bebida ou até mesmo da situação em que é consumida (Barros et al., 2012).

O álcool quando ingerido é absorvido na mucosa do estômago que, caso o indivíduo esteja em jejum, a absorção é mais rápida chegando mais rápido no cérebro e no fígado, acelerando a embriaguez (Silva, 1997). Quando chega no fígado, o 
álcool é metabolizado através de um processo chamado oxidação, onde a enzima citoplasmática álcool-desidrogenase (ADH) age convertendo-o em aldeído acético que é uma substância tóxica (Goodman, 1997).

Após a intoxicação por álcool, o sistema nervoso central é estimulado, causando euforia e comprometendo a memória, discernimento, coordenação motora e controle emocional, sendo importante ressaltar que, quando combinado com medicamentos antidepressivos, pode resultar em depressão respiratória e ser fatal (Golan, 2014).

O álcool tem por mecanismo de ação no organismo aumentar a ação do neurotransmissor GABA e do aminoácido glicina, impedir que os canais de cálcio se abram ativando os canais de potássio e inibir os receptores NMDA (glutamato) (Rang \& Dale, 2011).

Os medicamentos antidepressivos possuem efeitos adversos que devem ser levados em consideração e estão associados ao bloqueio de receptores: quando há o bloqueio de H1 (histamina) pode causar sedação, bloqueio de receptores aadrenérgicos é responsável pela hipotensão postural e o bloqueio dos receptores muscarínicos pode estimular a visão embaçada, boca seca e constipação (Rang \& Dale, 2011).

Este estudo justifica-se devido ao fato de diversos estudos apontarem para um relativo aumento do uso de drogas lícitas e ilícitas por universitários, e, ainda, uma expansão na quantidade de acadêmicos que fazem uso de medicamentos devido à diagnósticos de transtornos psicológicos tais como depressão e ansiedade. Nesta perspectiva, o principal objetivo desta pesquisa é verificar a relação entre a utilização de medicamentos antidepressivos e a ingestão de bebidas alcoólicas por estudantes universitários.

\section{Metodologia}

A metodologia adotada para a realização desta investigação foi a pesquisa bibliográfica de cunho qualitativo descritivo, que de acordo com Lakatos e Marconi (2003) possibilita que o pesquisador evidencie contribuições atuais, baseadas em estudos já desenvolvidos, viabilizando a transformação das compreensões e evidenciando à sociedade contribuições relativamente novas, que pouco a pouco vão se transformando.

Para isso, buscou-se os materiais através das plataformas de pesquisa acadêmica online Google Acadêmico, Scielo, Periódicos CAPES e Pubmed, por meio dos termos-chave: depressão em estudantes, interação entre antidepressivos e álcool e uso de álcool por estudantes, totalizando um quantitativo de 23 artigos. Destes, 10 foram excluídos da pesquisa por não compreenderem os objetivos da pesquisa com base na leitura do resumo das pesquisas e 3 foram descartados por não estarem disponíveis integralmente nas plataformas, impossibilitando a análise acurada dos resultados. Além disso, não foram selecionados resumos simples ou expandidos publicados em anais de eventos, apenas artigos científicos publicados em revistas, dissertações ou monografias relacionadas ao tema de pesquisa.

Dentre os critérios de inclusão, foram selecionados artigos compreendidos entre o período de janeiro de 2015 a julho de 2021, baseados em metodologias de revisão bibliográfica, exploratória descritiva, estudo de caso e relato de caso.

Sendo assim, totalizou-se a seleção e análise de 10 artigos, enquadrados nos critérios de inclusão à pesquisa, a fim de viabilizar uma melhor compreensão a respeito da interação entre fármacos antidepressivos e o uso de álcool por estudantes, bem como os comparativos do estudo.

\section{Resultados e Discussão}

O tratamento com intervenção farmacológica demanda um relativo cuidado por parte do paciente, sobretudo nos quadros de depressão, ansiedade e demais doenças psicológicas, posto que apenas o medicamento não traz resultados completamente eficazes, sendo necessário, portanto, uma transformação acerca da qualidade de vida, transformação de hábitos, psicoterapia e demais opções que auxiliem no mantenimento da saúde mental (Damasceno, et al., 2019). A Tabela 1 evidencia 
o posicionamento dos autores selecionados para a composição deste arcabouço teórico, acerca da utilização de medicamentos atrelada ao consumo de álcool, conforme os entendimentos.

Tabela 1. Principais considerações sobre o a interação medicamentosa e o álcool.

\begin{tabular}{|c|c|c|}
\hline Autor(es) & Metodologia & Principais resultados \\
\hline $\begin{array}{l}\text { Silva } \\
(2017)\end{array}$ & $\begin{array}{c}\text { Revisão sistemática de } \\
\text { bibliografia }\end{array}$ & $\begin{array}{l}\text { O autor considera a interação entre medicamentos e álcool muito perigosa, podendo acarretar efeitos } \\
\text { colaterais extremamente danosos à saúde do indivíduo. A potencialização do fármaco é evidenciada pela } \\
\text { utilização do álcool, aumentando expressivamente os efeitos ansiosos aos pacientes que estão em } \\
\text { tratamento. Além disso, pode causar complicações gastrointestinais, respiratórias, hipoglicemia e até } \\
\text { mesmo o óbito. }\end{array}$ \\
\hline $\begin{array}{l}\text { Silva et al. } \\
\quad(2021)\end{array}$ & $\begin{array}{l}\text { Pesquisa observacional e } \\
\text { transversal de abordagem } \\
\text { quantitativa }\end{array}$ & $\begin{array}{l}\text { Conforme os autores, a interação medicamentosa com o álcool não traz nenhum tipo de benefício ao } \\
\text { usuário, acarretando efeitos colaterais desastrosos. Faz-se essencial o acompanhamento } \\
\text { farmacoterapêutico, visando evitar a utilização indiscriminada de medicamentos, associada ao uso de } \\
\text { outras substâncias, que não agregam ao efeito benéfico do fármaco. }\end{array}$ \\
\hline $\begin{array}{l}\text { Oliveira Neto } \\
\text { (2018) }\end{array}$ & $\begin{array}{c}\text { Revisão bibliográfica } \\
\text { integrativa }\end{array}$ & $\begin{array}{l}\text { Para o autor, a combinação entre bebida alcoólica atrelada ao tratamento farmacológico resulta na perda } \\
\text { de eficácia dos compontes ativos do medicamento, proporcionando sérias problemáticas em relação à } \\
\text { segurança do paciente que está em tratamento. Entre os principais efeitos colaterais advindos dessa } \\
\text { prática associada, estão: hipoglicemia, cefaléia e antabuse. Evidencia-se a importância da disseminação } \\
\text { de informações para evitar e prevenir a má utilização dos fármacos. }\end{array}$ \\
\hline $\begin{array}{l}\text { Sodré et al. } \\
\text { (2021) }\end{array}$ & $\begin{array}{l}\text { Estudo transversal descritivo } \\
\text { e retrospectivo de abordagem } \\
\text { quantitativa }\end{array}$ & $\begin{array}{l}\text { Para os autores, as interações do medicamento com álcool resultam na ineficácia do tratamento } \\
\text { farmacológico, alterações negativas no organismo e interrupção do tratamento. É imprescindível a } \\
\text { atuação dos profissionais da saúde como indivíduos que podem auxiliar na conscientização dos } \\
\text { indivíduos, bem como realizar o acompanhamento farmacoterapêutico assegurando a eficiência do } \\
\text { tratamento. }\end{array}$ \\
\hline $\begin{array}{l}\text { Balen } \text { et al. } \\
\quad \text { (2017) }\end{array}$ & Estudo retrospectivo & $\begin{array}{l}\text { Os autores explicam que as interações medicamentosas entre dois ou mais fármacos e/ou substâncias que } \\
\text { visam potencializar o efeito do medicamento devem ser analisadas com cautela e evitadas ao máximo, } \\
\text { de modo a minimizar os riscos graves e moderados das interações, não trazendo ônus aos pacientes. } \\
\text { Outrossim, para que essa associação ocorra, deve haver a assistência de um profissional especializado, } \\
\text { sendo inadmissível a automedicação e associação não prescrita previamente. }\end{array}$ \\
\hline $\begin{array}{l}\text { Gotardelo } e t \\
\text { al. }(2015)\end{array}$ & Estudo transversal & $\begin{array}{l}\text { O estudo realizado pelos autores apontou para a importância da avaliação e orientação } \\
\text { farmacoterapêutica, principalmente pela associação dos fármacos com álcool. Dentre os } 272 \text { analisados, } \\
\text { apenas } 12 \text { indivíduos apresentaram reações adversas à algum tipo de associação. Contudo, é explícita a } \\
\text { importância de que a orientação seja realizada com os pacientes, minimizando os impactos desta prática. }\end{array}$ \\
\hline $\begin{array}{l}\text { Rodrigues et } \\
\text { al. (2018) }\end{array}$ & $\begin{array}{c}\text { Revisão sistemática de } \\
\text { bibliografia }\end{array}$ & $\begin{array}{l}\text { Na perspectiva dos autores, sozinho o álcool atua como um supressor do sistema nervoso, portanto } \\
\text { quando atrelado seu uso ao de algum fármaco, essa depressão é acentuada no organismo. Os efeitos } \\
\text { colaterais decorrentes dessa combinação são considerados graves e podem acarretar até mesmo na morta } \\
\text { do paciente, posto que essa combinação pode potencializar ou suprimir os efeitos do medicamento, } \\
\text { dependendo de cada indivíduo. Os principais órgãos afetados negativamente pela associação de } \\
\text { fármacos com o álcool são o fígado, o coração e os rins. }\end{array}$ \\
\hline $\begin{array}{l}\text { Medeiros } \\
\text { Filho } \text { et al. } \\
\quad(2018)\end{array}$ & Estudo transversal & $\begin{array}{l}\text { Cerca de } 26,5 \% \text { dos participantes da pesquisa admitiram fazer uso de álcool juntamente com outras } \\
\text { substâncias psicoativas. Outrossim, 94\% dos participantes reconheceram que a interação do } \\
\text { medicamento com o álcool pode ser danosa, acarretando efeitos colaterais de moderados a graves. }\end{array}$ \\
\hline $\begin{array}{l}\text { Bezerra } \\
(2019)\end{array}$ & $\begin{array}{l}\text { Pesquisa descritiva } \\
\text { quantitativa }\end{array}$ & $\begin{array}{l}\text { Evidencia que grande parte dos pacientes que fazem uso de fármacos antidepressivos reconhecem não } \\
\text { ser recomendado fazer o uso de álcool, contudo continuam associando as duas substâncias. É importante } \\
\text { a realização de estudos multidisciplinares que atinjam a estrutura educacional, mudando a percepção dos } \\
\text { indivíduos sobre a utilização correta e racional de medicamentos. }\end{array}$ \\
\hline $\begin{array}{l}\text { Sousa et al. } \\
\text { (2019) }\end{array}$ & $\begin{array}{c}\text { Revisão narrativa de } \\
\text { bibliografia }\end{array}$ & $\begin{array}{l}\text { Considera que a junção de medicamentos com álcool acarreta transformações - quase sempre negativas - } \\
\text { na absorção do fármaco. Também evidenciam a possibilidade de alterações, que resultam em efeitos } \\
\text { colaterais. }\end{array}$ \\
\hline
\end{tabular}

Fonte: Autores (2021).

De acordo com Souza et al. (2021) a maioria dos estudantes diagnosticados com transtornos psicológicos utiliza-se de fármacos para controle de seu estado emocional. Em sua pesquisa, cerca de $78 \%$ dos estudantes relataram a necessidade de intervenção medicamentosa após o ingresso na universidade. Os autores apontam para um relativo adoecimento da comunidade acadêmica devido aos percalços da graduação/pós-graduação, acentuado por situações de estresse, sobrecarga, pressão física e psicológica, entre outros fatores.

No entendimento de Silva et al. (2021) há uma relação intrínseca entre transtornos depressivos e ansiosos e o alcoolismo, o que reflete justamente na utilização integrada de fármacos e bebidas alcoólicas, sendo este último considerado 
como um refúgio e uma busca pela transformação, pelo esquecimento dos problemas e das dificuldades, inerentes à vida acadêmica, podendo a depressão e os demais transtornos psicológicos estar ligados à abstinência.

Sendo assim, evidencia-se a necessidade da atuação dos profissionais da farmácia e outras áreas multidisciplinares, atuantes na conscientização da população, na disseminação de informações, no acompanhamento do tratamento farmacoterapêutico do paciente, resguardando-lhe a plena segurança e o correto uso dos medicamentos (Silva et al., 2021; Souza et al., 2021; Sodré et al., 2019).

Pode-se considerar que a pressão e as preocupações inerentes ao âmbito acadêmico acarreta em problemas para os estudantes, que buscam saídas para minimizar o sofrimento psíquico, seja através de tratamento psicológico e psiquiátrico com intervenção medicamentosa, ou pelo uso de substâncias lícitas e ilícitas. De todo modo, é notável que a combinação de fármacos antidepressivos atreladas à ingestão de bebidas alcoólicas são extremamente prejudiciais à saúde do indivíduo e devem ser evitadas. Portanto, a intervenção profissional no acompanhamento dos pacientes e da conscientização da população acadêmica, de um modo geral, é de extrema necessidade.

\section{Conclusão}

Conclui-se diante do exposto, que uma parte dos estudantes universitários fazem o uso de medicamentos psicoterápicos, devido a quadros de estresse, traumas, sobrecarga física e mental, entre outros. Porém, grande parte destes acadêmicos utilizam-se de substâncias lícitas e ilícitas, das quais comumente cita-se a bebida alcóolica. Os estudos apontam para a ocorrência de diversos efeitos colaterais não benéficos quando atrelado o uso do antidepressivo com a bebida alcóolica, salientando a importância de que os profissionais de farmácia e áreas correlatas atuem estrategicamente, promovendo ações de conscientização, educação e acompanhamento dos pacientes, visando a efetividade do tratamento e a plena recuperação do indivíduo.

Faz-se necessária a continuidade - por parte da comunidade acadêmica - de pesquisar sobre essa temática, evidenciando as contribuições para a sociedade em geral, visando minimizar os impactos de práticas ineficazes e que apresentam riscos à integridade da saúde dos indivíduos.

Por fim, as próximas pesquisas acerca da temática poderão debruçar-se ainda mais sobre o assunto, baseando-se nos estudos que estão sendo desenvolvidos, verificando quais são os medicamentos mais utilizados pelos universitários e suas respectivas motivações, pois muito além de um problema na utilização irrestrita dos fármacos, é preciso compreender o que está motivando os acadêmicos a precisarem de tratamento psiquiátrico bem como abusar de substâncias tóxicas, como o álcool, entre outros fatores que ainda serão problemáticas pertinentes aos pesquisadores.

\section{Referências}

Balen, E., Giordani, F., Cano, M. F. F., Zonzini, F. H. T., Klein, K. A., Vieira, M. H. \& Mantovani, P. C. (2017). Interações medicamentosas potenciais entre medicamentos psicotrópicos dispensados. Jornal Brasileiro de Psiquiatria, 66(3), 172-177. http://doi.org/10.1590/0047-2085000000167.

Barros, C. L. V., Barros, D. A. C., Bernardes, M. J. C., Lima, W. V. \& Silva, L. C. S. (2012). A influência do convívio universitário na adesão ao alcoolismo. Revista Eletrônica do Curso de Pedagogia do Campus Jataí - UFG, 2(13), 1-5. https://doi.org/10.5216/rir.v2i13.22312.

Bezerra, K. (2019). Análise do perfil dos usuários de antidepressivos em uma farmácia pública no município de Turvo-PR. Monografia, Uniguaíra. http://200.150.122.211:8080/jspui/handle/23102004/133.

Cunha, M. F. E \& Gandini, R. C. (2009). Adesão e Não-Adesão ao Tratamento Farmacológico para Depressão. Psicologia: Teoria e Pesquisa, 25(3), 409-418. https://www.scielo.br/j/ptp/a/TNvpPTvvbbnYCmQVzYbWcpS/?lang=pt\&format=pdf.

Goodman, L S. (1997). As bases farmacológicas da terapêutica. Manole.

Gotardelo, D. R., Lopes, L. N., Meira, A. M. M., Costa, C. K. M., Masson, E. R., Fonseca, L. S., Toledo, V. N., Faioli, M. A. \& Andrade, R. B. L. (2015). Consumo de álcool e interações álcool-drogas entre idosos atendidos na Estratégia Saúde da Família. Revista Médica de Minas Gerais, 25(3), 363-368. http://doi.org/10.5935/2238-3182.20150071. 
Research, Society and Development, v. 10, n. 15, e190101522441, 2021

(CC BY 4.0) | ISSN 2525-3409 | DOI: http://dx.doi.org/10.33448/rsd-v10i15.22441

Masur, J., \& Monteiro, M. G. (1983). Validation of the "CAGE" alcoholism screening test in a Brazilian psychiatric inpatient hospital setting. Brazil Journal Medicine Biology Research, 16(1), 215-218.

Medeiros Filho, J. S. A., Azevedo, D. M., Pinto, T. R., \& Silva, G. W. S. (2018). Uso de psicofármacos na atenção primária à saúde. Revista Brasileira em Promoção da Saúde, 31(3), 1-12. http://doi.org/10.5020/18061230.2018.7670.

Neves, A. L. A. (2015). Tratamento farmacológico da depressão. Dissertação (Mestrado) - Universidade Fernando Pessoa.

Oliveira Neto, A. C. (2018). Interação álcool x medicamento: uma revisão da literatura. Monografia. Cuité - Universidade Federal de Campina Grande.

Rang, H. P., Dale, M. M., Ritter, J. M., Flower, R. J. \& Henderson, G. (2011). Farmacologia. Elsevier.

Rodrigues, M. M. S., Anjos, I. P., Santos, M. V. P. \& Dourado, K. M. C. (2018). Combinação de medicmentos e álcool e seus efeitos adversos no corpo humano. Candombá, 14(1), 1-9. http://web.unijorge.edu.br/sites/candomba/teste/pdf/artigos/2018/combinacao.pdf.

Silva, A. O., Barbosa, A. A., Cunha, A. P. S., Rolim, I. A. A., Santos, R. F., Borges, J. M. P. \& Lemos, G. S. (2021). Interações potenciais entre medicamentos e medicamentos-álcool em pacientes. Research, Society and Development, 10(9), 1-15. https://doi.org/10.33448/rsd-v10i9.17697.

Silva, P. (1997). Farmacologia. Manole.

Silva, S. V. L. A interação do álcool com medicamentos e seus efeitos no organismo. (2017). Monografia. Ariquemes - Faculdade de Educação e Meio Ambiente. https://repositorio.faema.edu.br/handle/123456789/1255.

Sodré, M. L. G., Ferreira, A. S., Borges, M. C. F., Marinho, S. F., Fernandes, M. A., Barros, C. S., Belfort, I. K. P., \& Monteiro, S. C. M. (2021). Potenciais interações medicamentosas no Centro de Atenção Psicossocial Álcool e Drogas em uma capital do Nordeste brasileiro. Research, Society and Development, 10(9), 1-11. http://dx.doi.org/10.33448/rsd-v10i9.17714. 